\title{
Up-regulation of FoxN4 Expression in Adult Spinal Cord After Injury
}

\author{
Xiangdong Chen • Yu Yao • Junjie Guan • \\ Xiaoqing Chen $\cdot$ Feng Zhang
}

Received: 1 July 2013 / Accepted: 28 October 2013 /Published online: 12 November 2013

(C) Springer Science+Business Media New York 2013

\begin{abstract}
FoxN4 (forkhead box N4), which is a transcription factor involved in developing spinal cord and spinal neurogenesis, implied important roles in the central nervous system (CNS). However, its expression and function in the adult CNS lesion are still unclear. In this study, we established a spinal cord injury (SCI) model in adult rats and investigated the expression of FoxN4 in the spinal cord. Western blot analysis revealed that FoxN4 was present in normal spinal cord. It gradually increased, peaked at day 3 after SCI, and then decreased during the following days. Immunohistochemistry further confirmed that FoxN4 was expressed at low levels in gray and white matters in normal condition and increased after SCI. Double immunofluorescence staining showed that FoxN4 is located on neurons and astrocytes, and FoxN4 expression was increased progressively in reactive astrocytes within the vicinity of the lesion, predominately in the white matter. In addition, almost all FoxN4-positive cells also expressed nestin or PCNA. Our data suggested that FoxN4 might play important roles in CNS pathophysiology after SCI.
\end{abstract}

Keywords FoxN4 $\cdot$ Spinal cord injury $\cdot$ Astrocytes $\cdot$ PCNA

\section{Introduction}

Spinal cord injury (SCI) is a common neurological disorder that causes death in children and adults and results in a significant society burden all over the world (Sekhon and Fehlings 2001; McDonald and Sadowsky 2002). The injured

Xiangdong Chen and Yu Yao contributed equally to this work.

X. Chen $\cdot$ Y. Yao $\cdot$ J. Guan $\cdot$ X. Chen $\cdot$ F. Zhang $(\bowtie)$

Department of Spine Surgery, Affiliated Hospital of Nantong University, Nantong 226001, Jiangsu, People's Republic of China

e-mail: ntfyzhangfeng@163.com spinal cord makes axons fail to regenerate, which limits motor and autonomic recovery and leads to long-term morbidity (Miller et al. 2004; Samanta and Kessler 2004; See et al. 2004; Chen and Panchision 2007). The pathology associated with SCI often involves complex cascade of physiological and biochemical mechanisms, which eventually cause neuronal apoptosis and astrocyte proliferation (Choi et al. 2010; Kwon et al. 2004; White et al. 2010). Astrocytes are the major glial cell population mainly responsible for the central nervous system (CNS) homeostasis (Rivieccio et al. 2005). The astrocyte proliferation and astrogliosis after CNS injury result in the formation of dense astrocytic scar which provide physical and biochemical barrier to plasticity and regeneration, and give multiple inhibitory factors which affect functional recovery following SCI (Davies et al. 1996; Silver and Miller 2004). Thus, the study of molecular and cellular mechanisms in spinal cord recovery after SCI and identification of the involved proteins will be helpful to the recovery of the injured spinal cord.

The forkhead/winged helix family is a large family of transcriptional regulators, which involved in a wide range of biological processes, such as proliferation, apoptosis, development, differentiation, migration, and invasion (Laoukili et al. 2005; Koo, Muir and Lam 2012). Among these characteristics, forkhead family seems particularly important in cell differentiation, developmental processes, and neurogenesis in the CNS (Filosa et al. 1997; Labosky et al. 1997; Herrera et al. 2004; Vargha-Khadem et al. 2005), such as FoxJ1, which is required for activating a subset of astrocytes that participate in neurogenesis and gliogenesis in spinal cord injury and in response to stroke (Jacquet et al. 2009; Meletis et al. 2008; Carlen et al. 2009). FoxN4 is a member of the forkhead/ winged helix family, which involved in the specification of amacrine and horizontal cells during retinogenesis ( $\mathrm{Li}$ et al. 2004). It is expressed in retina, ventral hindbrain, spinal cord, and dorsal midbrain during neural development. FoxN4 
expression is associated with proliferating progenitor cells in the retina. FoxN4 modulates the proliferation and destination of retinal progenitors by activating Dll4-notch signaling pathway (Luo et al. 2012). Importantly, Gouge et al. demonstrated that FoxN4 was expressed in the developing spinal cord but a role has yet to be clarified during spinal neurogenesis (Gouge et al. 2001) in the CNS. We hypothesized that FoxN4 may be involved in the pathophysiological and biochemical progression after SCI, which was associated with the outcome of neurogenesis after injury.

In the present study, we reported the expression and distribution of FoxN4 after spinal cord injury in rat for the first time. Our research is conducted to give better insights into the physiologic functions of FoxN4 and its molecular mechanisms underlying astrocyte proliferation in neurogenesis after SCI in spinal cord.

\section{Materials and Methods}

\section{Animals and Surgery}

Male Sprague-Dawley rats $(n=48)$ with an average body weight of $250 \mathrm{~g}(220-275 \mathrm{~g})$ were used in this study. The rats were anesthetized with pentobarbital (50 mg/kg i.p.). Contusion injuries were performed at spinal segment $\mathrm{T} 9$ using the NYU impactor (Gruner 1992). A 10-g, 2.0-mm-diameter rod was released from $10-\mathrm{cm}$ height onto the exposed spinal cord $(n=42)$. After SCI, the overlying muscles and skin were closed in layers with 4-0 silk sutures and staples, respectively. Animals were allowed to recover on a $30{ }^{\circ} \mathrm{C}$ heating pad. Postoperative treatments included saline $(2.0 \mathrm{cc}$, s.c.) for rehydration and Baytril $(0.3 \mathrm{cc}, 22.7 \mathrm{mg} / \mathrm{ml}$, s.c., twice daily) to prevent urinary tract infection. Bladders were manually expressed twice daily until reflex bladder emptying returned. Animals were sacrificed at 6 h, 12 h, 1 day, 3 days, 5 days, 7 days, and 14 days after injury. Sham-operated animals $(n=$ 6) were served as noninjured controls. All surgical interventions and postoperative animal care were carried out in accordance with the Guide for the Care and Use of Laboratory Animals and were approved by the Chinese National Committee to the Use of Experimental Animals for Medical Purposes, Jiangsu Branch. All efforts were made to minimize the number of animals used and their suffering.

\section{Western Blot Analysis}

To obtain samples for Western blot, the sham-operated or injured spinal cords were excised and snap frozen at $-70{ }^{\circ} \mathrm{C}$ until use. The portion of spinal cord extending $5 \mathrm{~mm}$ rostral and $5 \mathrm{~mm}$ caudal to the injury epicenter was immediately removed. To prepare lysates, frozen spinal cord samples were minced with eye scissors in ice. The samples were then homogenized in lysis buffer (1\% NP-40, $50 \mathrm{mmol} / 1$ Tris, pH 7.5, 5 mmol/1 EDTA, $1 \%$ SDS, $1 \%$ sodium deoxycholate, $1 \%$ Triton X-100, $1 \mathrm{mmol} / \mathrm{l}$ PMSF, $10 \mathrm{mg} / \mathrm{ml}$ aprotinin, and $1 \mathrm{mg} / \mathrm{ml}$ leupeptin) and clarified by centrifuging for $20 \mathrm{~min}$ in centrifuge at $4{ }^{\circ} \mathrm{C}$. After measured protein concentration with the Bradford assay (Bio-Rad), the supernatant (50 $\mathrm{mg}$ of protein) was subjected to SDS-polyacrylamide gel electrophoresis (PAGE). The separated proteins were transferred to a polyvinylidine difluoridemembrane (Millipore) by a transfer apparatus at $350 \mathrm{~mA}$ for $2.5 \mathrm{~h}$. The membrane was then blocked with $5 \%$ nonfat milk and incubated with primary antibody against FoxN4 (anti-rabbit, 1:500; Santa Cruz) or GAPDH (anti-rabbit, 1:1,000; Santa Cruz). After incubating with an anti-mouse or anti-rabbit horseradish peroxidaseconjugated secondary antibody, protein was visualized by enhanced chemiluminescence system (ECL, Pierce Company, USA).

Immunohistochemistry

After defined survival times, sham-operated and injured rats were terminally anesthetized and perfused through the ascending aorta with saline, followed by $4 \%$ paraformaldehyde. After perfusion, the sham-operated and injured spinal cords were removed and post-fixed in the same fixative for $3 \mathrm{~h}$ and then replaced with $20 \%$ sucrose for $2-3$ days, following $30 \%$ sucrose for 2-3 days. After treatment with sucrose solutions, the tissues were embedded in O.T.C. compound. Then, $8-\mu \mathrm{m}$ frozen cross-sections at two spinal cord levels $(2 \mathrm{~mm}$ rostral and caudal to the epicenter of injury) were prepared and examined. All of the sections were blocked with $10 \%$ goat serum with $0.3 \%$ Triton X-100 and $1 \%(\mathrm{w} / \mathrm{v})$ bovine serum albumin (BSA) for $2 \mathrm{~h}$ at room temperature (RT) and incubated with anti-FoxN4 antibody (anti-rabbit, 1:100; Santa Cruz) at $4{ }^{\circ} \mathrm{C}$ overnight, followed by incubation in biotinylated secondary antibody (Vector Laboratories, Burlingame, CA). Staining was visualized with DAB (Diaminobenzidin, Vector Laboratories). Cells with strong or moderate brown staining were counted as positive, cells with no staining were counted as negative, and cells with weak staining were scored separately. For double immunofluorescent staining, sections were firstly blocked with $10 \%$ normal serum blocking solution species the same as the secondary antibody, containing $3 \%$ BSA and $0.1 \%$ Triton X-100 and $0.05 \%$ Tween-20 $2 \mathrm{~h}$ at RT in order to avoid nonspecific staining. Then, the sections were incubated with both rabbit polyclonal primary antibodies for FoxN4 (1:200; Santa Cruz) and mouse monoclonal antibody for PCNA (1:100; Santa Cruz) or mouse monoclonal antibody for nestin (1:100; Santa Cruz) or different markers as follows: NeuN (neuron marker, 1:600; Millipore) and GFAP (astrocyte marker, 1:200; Sigma). Briefly, sections were incubated with both primary antibodies at $4{ }^{\circ} \mathrm{C}$ overnight, followed by a mixture of $\mathrm{Cy} 3$ - and $\mathrm{Cy} 2$-conjugated secondary antibodies 
for $2 \mathrm{~h}$ at $4{ }^{\circ} \mathrm{C}$. The stained sections were examined with a Leica fluorescence microscope (Leica, DM 5000B; Leica CTR 5000; Germany).

\section{Quantitative Analysis}

Cell quantification in the spinal cord was performed according to the method described by Shen et al. (Shen et al. 2008a) and Zhang et al. (Zhang et al. 2013). The number of FoxN4positive cells in the spinal cord $2 \mathrm{~mm}$ caudal to the epicenter was counted in a $500 \times 500 \mu \mathrm{m}$ measuring frame. For each animal, a measure was taken in a section through the dorsal horn, the lateral funiculus, and the ventral horn. To avoid counting the same cell in more than one section, we counted every fifth section $(40 \mu \mathrm{m}$ apart). The cell counts in the three or four adjacent sections were then used to determine the total number of FoxN4-positive cells per squared millimeter. Cells double labeled for FoxN4 and the other phenotypic markers used in the experiment were quantified. Sections were double labeled for FoxN4 and for NeuN and GFAP. To identify the proportion of each phenotype-specific marker-positive cells expressing FoxN4, a minimum of 200 phenotype-specific marker-positive cells was counted in both of the gray matter and white matter on each section, with the exception that only gray matter was conducted for NeuN. Then, double-labeled cells for FoxN4 and the phenotype-specific markers were recorded. Two or three adjacent sections per animal were sampled $2 \mathrm{~mm}$ to the epicenter.

\section{$\mathbf{A}$}

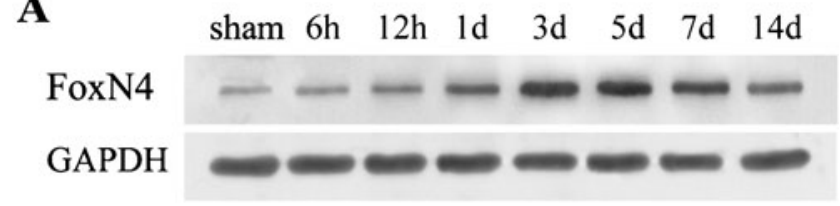

B

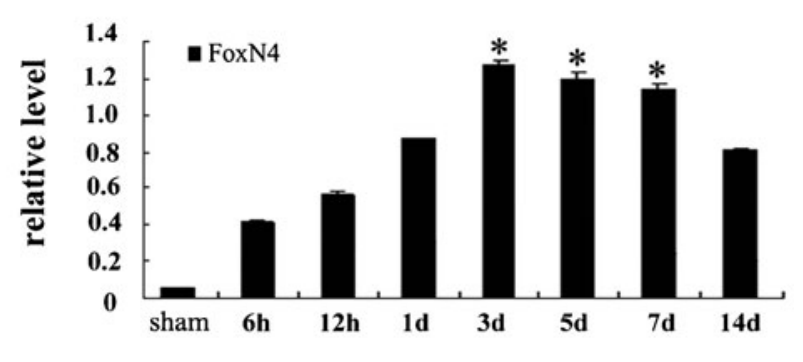

time after SCI

Fig. 1 Expression of the FoxN4 protein after SCI. a Spinal cord segments were extracted and prepared for Western blotting at various times after SCI using anti-FoxN4 and anti-GAPDH antibodies. Depicted is a representative blot from three independent experiments with similar results. b Quantitative analysis of three Western blots (normalized to GAPDH) using the NIH Image Analysis System graphically illustrated an increase in FoxN4 level after SCI in a time-dependent manner. Data in relative density are expressed as mean \pm SD. $* P<0.05$ versus control group

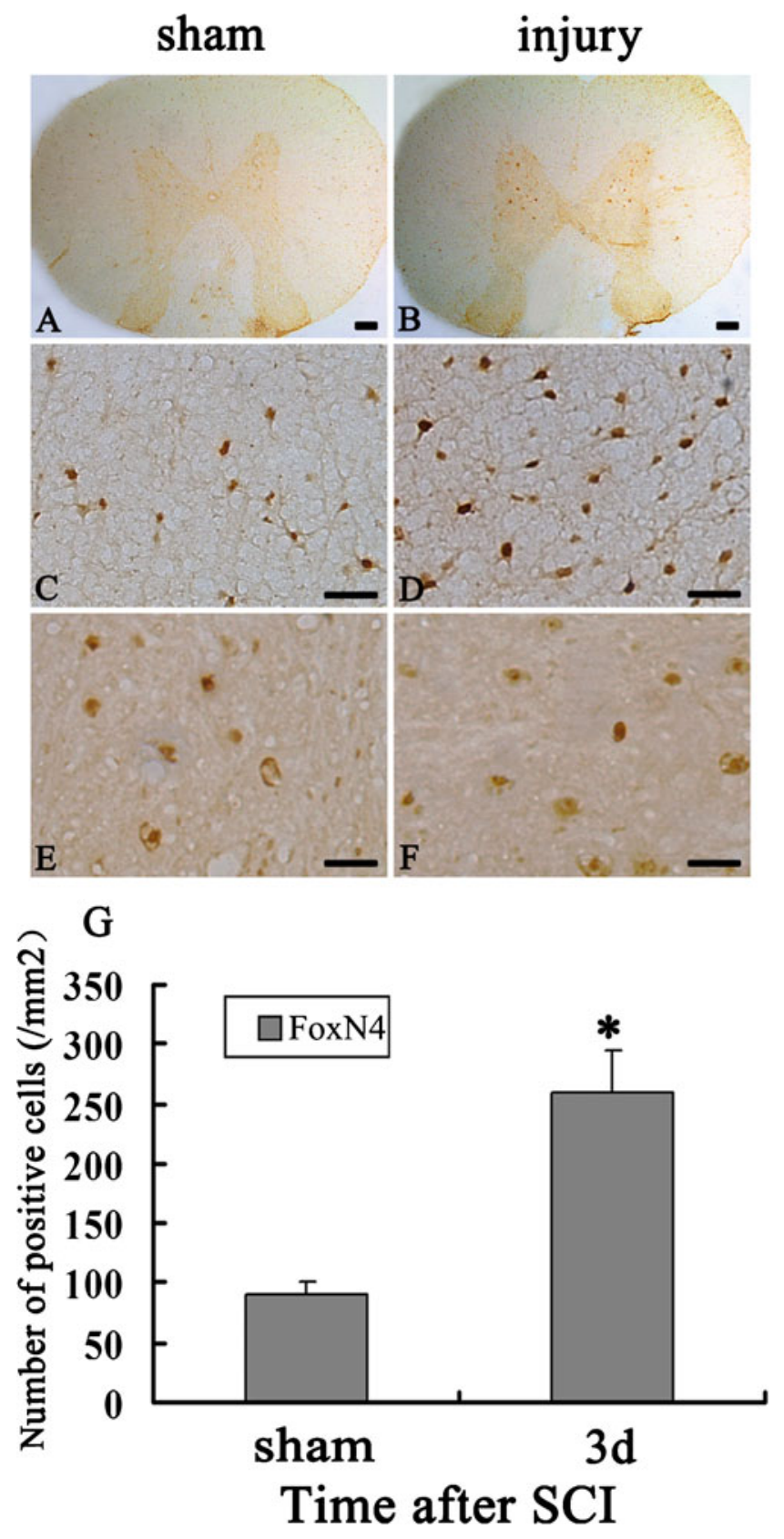

Fig. 2 Immunohistochemical expression of FoxN4 in adult rat spinal cord $2 \mathrm{~mm}$ rostral to epicenter. Low-power views of cross-sections immunostained with antibody specific for FoxN4 in control spinal cord (a) and day 3 after injury (b). Higher-power views in the white matter (c) and ventral horn (e) of control spinal cord. FoxN4 staining was increased in the ventral horn (f) and white matter (d) at day 3 after injury. Scale bars $200 \mu \mathrm{m}(\mathbf{a}$ and $\mathbf{b})$ and $20 \mu \mathrm{m}(\mathbf{c}-\mathbf{f})$. g Quantitative analysis of FoxN4positive cells $/ \mathrm{mm}^{2}$ between control and day 3 after SCI. Asterisk (*) indicates significant difference at $P<0.05$ compared with control. Error bars represent SEM

\section{Statistical Analysis}

All data were analyzed with Stata 7.0 statistical software. All values are expressed as means \pm SEM. One-way ANOVA followed by the Tukey's post hoc multiple comparison tests 
Fig. 3 Double

immunofluorescence staining for FoxN4 and cells markers in adult spinal cord. In the adult rat spinal cord $2 \mathrm{~mm}$ to epicenter at day 3 after SCI, horizontal sections labeled with FoxN4 (red) and different phenotype-specific markers (green), including NeuN (a-h), GFAP (i-p), and the colocalization of FoxN4 with different phenotype-specific markers, as shown in the control ventral horn $(\mathbf{a}-\mathbf{h})$ and white matter (i-p). q Quantitative analysis of different phenotypespecific markers positive cells expressing FoxN4 (\%) in control spinal cord and day 3 after SCI.

The changes of FoxN4 expression after SCI were prominent in astrocytes. Asterisk (*) indicates significant difference at $P<0.05$ compared with control. Error bars represent SEM. Scale bars: $20 \mu \mathrm{m}$

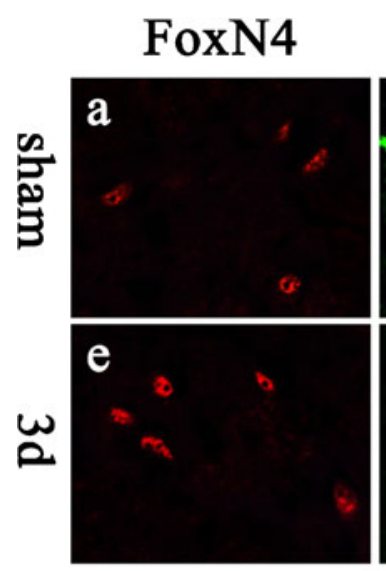

$\mathrm{NeuN}$

DAPI
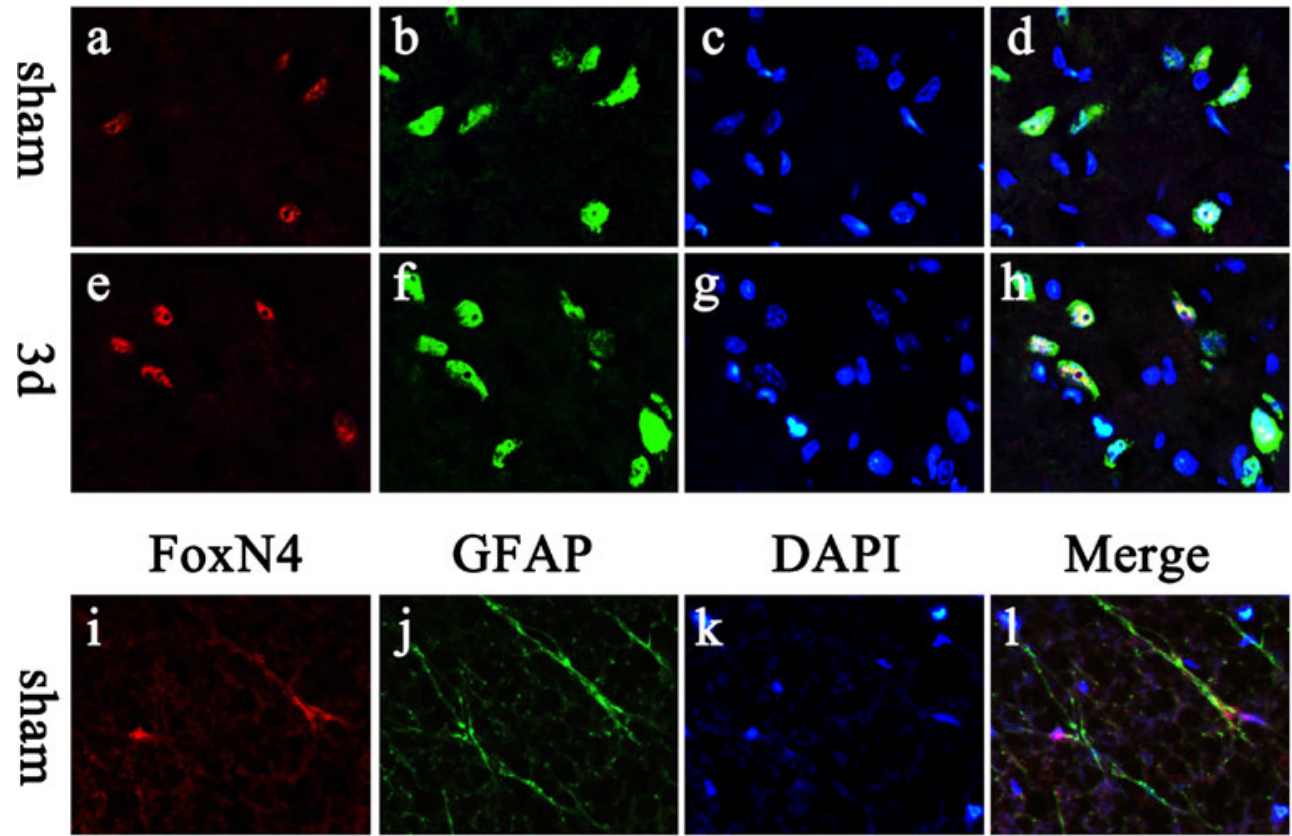

DAPI

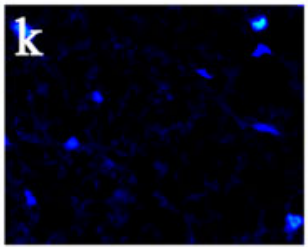

Merge
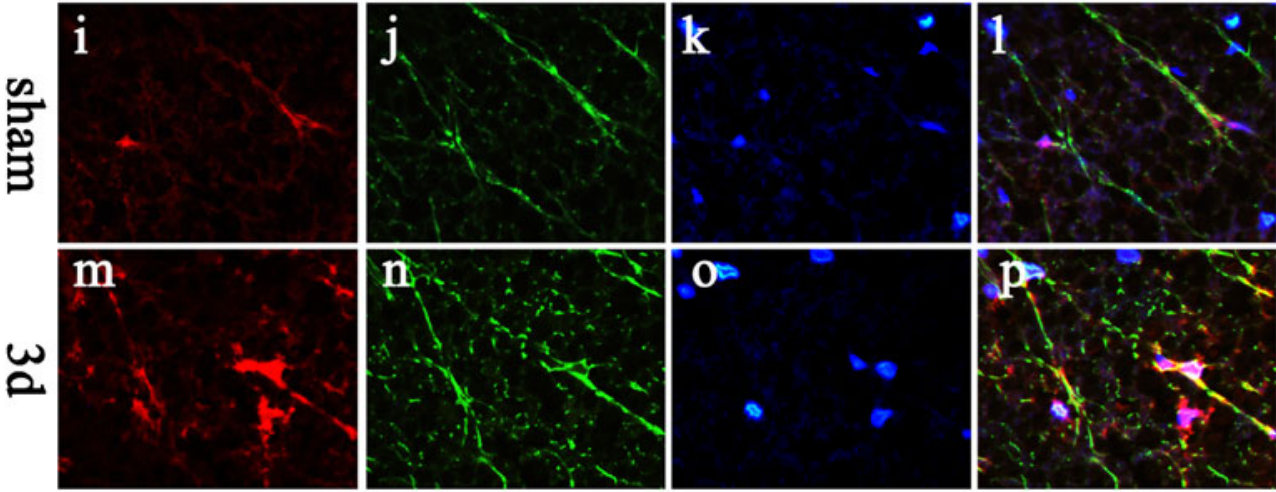

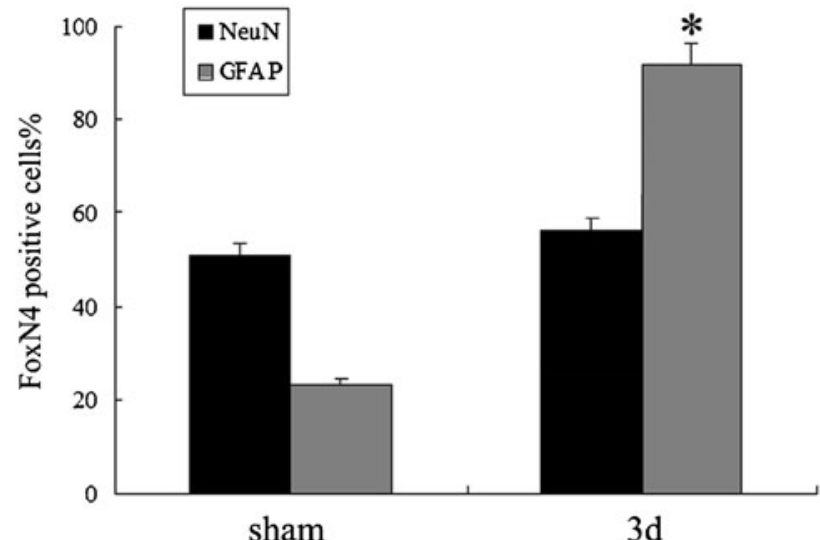

was used for statistical analysis. $P$ values less than 0.05 were considered statistically significant. Each experiment consisted of at least three replicates per condition.

\section{Results}

The Expression Profiles of FoxN4 Following Spinal Cord Injury by Western Blot Analysis

Western blot was performed to investigate the expression of FoxN4 after SCI. FoxN4 protein level was low in control spinal cords, rose at $6 \mathrm{~h}$ after SCI and reached a peak at 3 days $(P<0.05)$, and then gradually decreased to normal level (Fig. 1). This result demonstrated that FoxN4 might be involved in the process of SCI in a time-dependent manner.

\section{The Staining Changes of FoxN4 in Spinal Cord}

To identify the distribution of FoxN4 after SCI, we performed immunohistochemistry with anti-FoxN4 rabbit polyclonal antibody. As Western blot analysis indicated, FoxN4 has the highest protein expression at day 3 after SCI, so we chose day 3 as the time point of injury immunohistochemistry. In the 
spinal cord $2 \mathrm{~mm}$ caudal to epicenter, FoxN4 was widely expressed in both the ventral horn and the white matter of the spinal cord (Fig. 2a-f), regardless of whether the animals were control or injured. FoxN4 was largely up-regulated in the nuclei after injury. In addition, the number of FoxN4-positive cells measured between control spinal cords and spinal cords 3 days after injury was consistent with Western blot results (Fig. 2g).

The Up-regulation of FoxN4 Was Predominant in Astrocytes, Which Were Largely Proliferated After Injury

To further confirm the cell types expressing FoxN4, double labeling immunofluorescent staining was performed with two cell-specific markers: NeuN (marker of neuron, Fig. 3a-h) and GFAP (marker of astrocyte, Fig. 3i-p). To identify the proportion of neurons and astrocytes expressing FoxN4, a minimum of 200 neurons and astrocytes was counted between control spinal cords and spinal cords 3 days after SCI (as described in"Materials and Methods"). FoxN4 was expressed in both neurons and astrocytes, with relatively low levels of expression in control spinal cords. At 3 days after injury, FoxN4 expression was significantly increased in astrocytes $(P<0.05)$ compared to control spinal cords (Fig. 3q).

FoxN4 Colocalization with PCNA and Nestin in Injured Spinal Cords

Double labeling immunofluorescence staining not only showed FoxN4 colocalization with astrocytes (Fig.3g-1) but also revealed that the number of astrocytes increased both in size and in complexity of their processes in response to SCI. Thus, we speculated that FoxN4 may be related to astrocyte proliferation in the spinal cord after SCI. Studies have proved that at day 3 post-injury, most reactive astrocytes were PCNA positive (Shen et al. 2008b; Zhao et al. 2011; Zhang et al. 2013). And other studies have reported that reactive astrocytes appear as strong expression of GFAP and nestin after SCI (Eng 1985). In this study, cell proliferation evaluated by PCNA appeared in many FoxN4-expressing cells at day 3 after injury (Fig. 4a-c). Importantly, we also found that many FoxN4-positive cells also express nestin (Fig. 4d-f). Collectively, the aforementioned evidences demonstrate the hypothesis that up-regulation of FoxN4 in the spinal cord was concomitant with astrocyte proliferation and further suggest that FoxN4 may be required for the differentiation of adult neural stem cell which participates in neurogenesis and gives rise to astrocytes after SCI.

\section{Discussion}

Astrocytes are the main cell type involved in spinal cord injury and becoming reactive and increasing in size that lead to a reactive phenotype capable of mediating wound healing following central nervous system injury (Wu et al. 2012; Yin et al. 2012). Astrocytes play very important roles in the homeostatic functions that directly influence neuronal survival, tissue integrity, and functional outcome after SCI, and they often undergo changes in gene expression, cellular hypertrophy, cell activation, and proliferation. Over-activation of astrocytes may lead to formation of glial scar and secretion of other chemicals to prevent regeneration after spinal cord injury (Wu et al. 2012; Bao et al. 2012). After SCI, reactive astrocytes appear as strong expression of intermediate filaments, such as GFAP and nestin (Eng 1985). In the injured
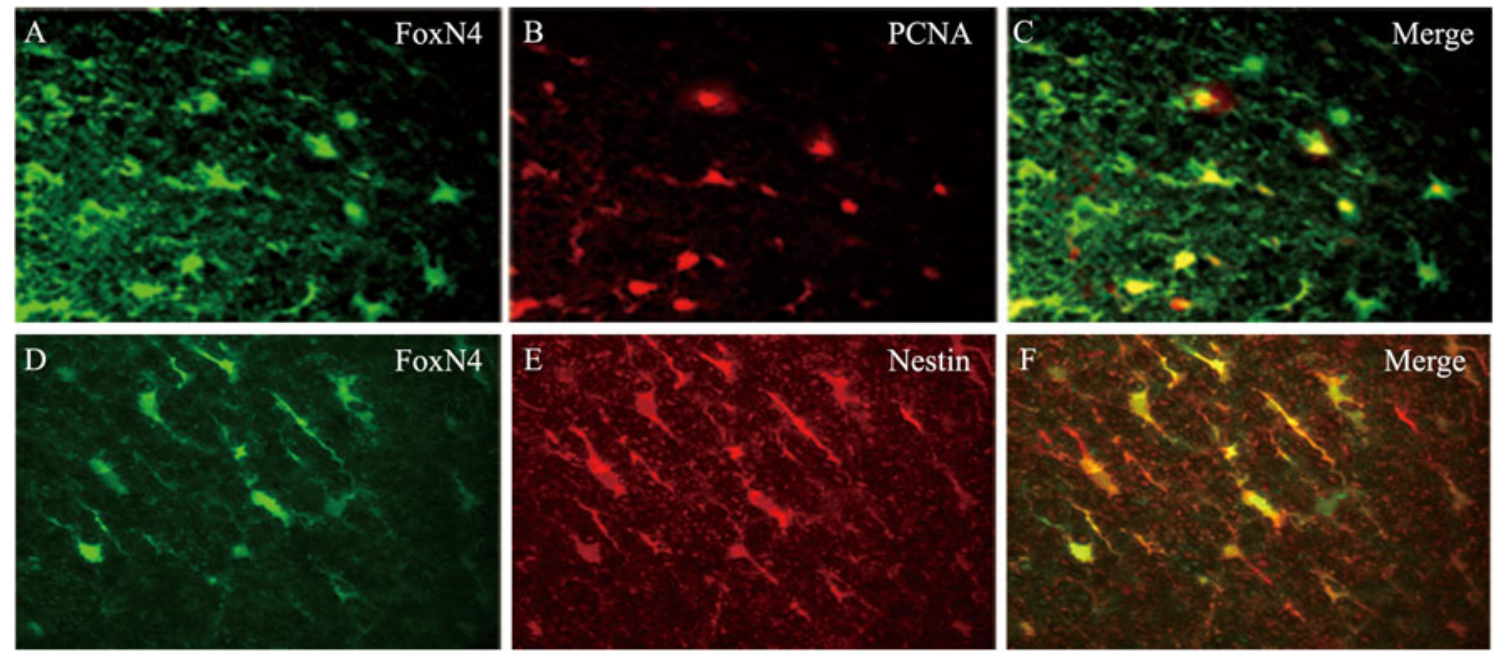

Fig. 4 FoxN4 colocalization with PCNA and nestin in injured spinal cords. In adult rat spinal cord $2 \mathrm{~mm}$ rostral to epicenter at day 3 after SCI, horizontal sections labeled with PCNA and FoxN4, the colocalization of
PCNA with FoxN4, are shown in the white matter $(\mathbf{a}-\mathbf{c})$. FoxN4 colocalization with nestin is also shown $(\mathbf{d}-\mathbf{f})$. Scale bars: $20 \mu \mathrm{m}$ 
CNS, reactive astrocytes lead to both beneficial and detrimental effects on surrounding cells. Reactive astrocytes also play a crucial role in wound healing and functional recovery after SCI. At the subacute phase, astrocytes migrate to compact the lesion, presumably secluding the inflammatory cells to prevent them from spreading into the parenchyma of the spinal cord (Okada et al. 2006). The reactive astrocytes are ubiquitously present at all sites of CNS pathologies; however, the molecular mechanisms, functions, and effects of reactive astrocytes are poorly understood (Vartak-Sharma and Ghorpade 2012). In this study, following a physical damage of spinal cord, reactive astrocytes in the vicinity of the damage site show increased FoxN4 immunoreactivity. And the observation of FoxN4 colocalizated with GFAP and PCNA in the white matter after spinal cord injury indicated that the upregulated expression of FoxN4 would induce astrocyte proliferation and may be deeply involved in tissue repair and functional recovery after SCI.

Neural stem/progenitor cells play important roles during neurogenesis after SCI (McDonald et al. 1999; Pfeifer et al. 2004; Picard-Riera et al. 2004; Horky et al. 2006). Studies revealed that neural stem/progenitor cells existed around the central canal of spinal cord in adult mammalian, which proliferated and migrated from the region of the central canal to white matter after SCI, and then terminally differentiated into astrocytes (Frisen et al. 1995; Weiss et al. 1996; Mothe and Tator 2005). It has been reported that reactive astrocytes in injured adult rat spinal cord may acquire the potential of neural stem/progenitor cells (Lang et al. 2004). The injured zone appears a lot of nestin and GFAP double labeled positive cells, and Nestin has been defined only expressed in cytoplasm of neuroepithelial precursor cells (Lendahl et al. 1990; Sergent-Tanguy et al. 2006), implied neural stem/ progenitor cells had migrated and reactive astrocytes may have the potential of neural stem/ progenitor cells after SCI. The finding that neurogenesis induced by injury shows a role in the process of repairing mammalian spinal cord. Studies have displayed that FoxN4 was expressed in a subset of $\mathrm{p} 2$ progenitors in spinal cord and played an important role during spinal neurogenesis (Gouge et al. 2001; Li et al. 2005). We find that almost all FoxN4-positive cells also express nestin in white matter after SCI. Combining these results, it suggests that FoxN4 may be required for the differentiation of the cells acting as adult neural stem cells which participate in neurogenesis and give rise to astrocytes after SCI. These cells may migrate to the lesion region and compensate for the loss of neuronal function by stimulation of injury in SCI.

Our study revealed that the expression of FoxN4 was significantly increased in the rat spinal cord after injury, which supported the concepts that FoxN4 may be involved in the physiological and the pathological processes in injured spinal cord. Our experiment may provide a novel strategy for treatment of CNS trauma in the field of neurogenesis. Further studies are needed to confirm the inherent mechanisms of the role of FoxN4 after SCI.

\section{References}

Bao Y, Qin L, Kim E, Bhosle S, Guo H, Febbraio M, Haskew-Layton RE, Ratan R, Cho S (2012) CD36 is involved in astrocyte activation and astroglial scar formation. J Cereb Blood Flow Metab 32:1567-1577

Carlen MK, Meletis C, Goritz V et al (2009) Forebrain ependymal cells are notch-dependent and generate neuroblasts and astrocytes after stroke. Nat Neurosci 12:259-267

Chen HL, Panchision DM (2007) Concise review: bone morphogenetic protein pleiotropism in neural stem cells and their derivatives-alternative pathways, convergent signals. Stem Cells 25:63-68

Choi DC, Lee JY, Moon YJ et al (2010) Acupuncture-mediated inhibition of inflammation facilitates significant functional recovery after spinal cord injury. Neurobiol Dis 39:272-282

Davies SJ, Field PM, Raisman G (1996) Regeneration of cut adult axons fails even in the presence of continuous aligned glial pathways. Exp Neurol 142:203-216

Eng LF (1985) Glial fibrillary acidic protein (GFAP): the major protein of glial intermediate filaments in differentiated astrocytes. J Neuroimmunol 8:203-214

Filosa S, Rivera-Perez JA, Gomez AP et al (1997) Goosecoid and HNF3beta genetically interact to regulate neural tube patterning during mouse embryogenesis. Development 124:2843-2854

Frisen J, Johansson CB, Torok C et al (1995) Rapid, widespread, and longlasting induction of nestin contributes to the generation of glial scar tissue after CNS injury. J Cell Biol 131:453-464

Gouge A, Holt J, Hardy AP et al (2001) Foxn4-a new member of the forkhead gene family is expressed in the retina. Mech Dev 107:203206

Gruner JA (1992) A monitored contusion model of spinal cord injury in the rat. J Neurotrauma 9:123-126, discussion 16-8

Herrera E, Marcus R, Li S et al (2004) Foxd1 is required for proper formation of the optic chiasm. Development 131:5727-5739

Horky LL, Galimi F, Gage FH, Horner PJ (2006) Fate of endogenous stem/progenitor cells following spinal cord injury. J Comp Neurol 498:525-538

Jacquet BV, Salinas-Mondragon R, Liang H et al (2009) FoxJ1dependent gene expression is required for differentiation of radial glia into ependymal cells and a subset of astrocytes in the postnatal brain. Development 136:4021-4031

Koo CY, Muir KW, Lam EW (2012) FOXM1: from cancer initiation to progression and treatment. Biochim Biophys Acta 1819:28-37

Kwon BK, Tetzlaff W, Grauer JN et al (2004) Pathophysiology and pharmacologic treatment of acute spinal cord injury. Spine J 4: 451-464

Labosky PA, Winnier GE, Jetton TL et al (1997) The winged helix gene, $\mathrm{Mf3}$, is required for normal development of the diencephalon and midbrain, postnatal growth and the milk-ejection reflex. Development 124:1263-1274

Lang B, Liu HL, Liu R et al (2004) Astrocytes in injured adult rat spinal cord may acquire the potential of neural stem cells. Neuroscience 128:775-783

Laoukili J, Kooistra MR, Bras A et al (2005) FoxM1 is required for execution of the mitotic programme and chromosome stability. Nat Cell Biol 7:126-136

Lendahl U, Zimmerman LB, McKay RD (1990) CNS stem cells express a new class of intermediate filament protein. Cell 60:585-595 
Li S, Misra K, Matise MP, Xiang M (2005) Foxn4 acts synergistically with Mash1 to specify subtype identity of V2 interneurons in the spinal cord. Proc Natl Acad Sci U S A 102:10688-10693

Li S, Mo Z, Yang X et al (2004) Foxn4 controls the genesis of amacrine and horizontal cells by retinal progenitors. Neuron 43:795-807

Luo H, Jin K, Xie Z et al (2012) Forkhead box N4 (Foxn4) activates Dll4Notch signaling to suppress photoreceptor cell fates of early retinal progenitors. Proc Natl Acad Sci U S A 109:E553-E562

McDonald JW, Liu XZ, Qu Y et al (1999) Transplanted embryonic stem cells survive, differentiate and promote recovery in injured rat spinal cord. Nat Med 5:1410-1412

McDonald JW, Sadowsky C (2002) Spinal-cord injury. Lancet 359:417425

Meletis K, Barnabe-Heider F, Carlen M et al (2008) Spinal cord injury reveals multilineage differentiation of ependymal cells. PLoS Biol 6: e182

Miller RH, Dinsio K, Wang R et al (2004) Patterning of spinal cord oligodendrocyte development by dorsally derived BMP4. J Neurosci Res 76:9-19

Mothe AJ, Tator CH (2005) Proliferation, migration, and differentiation of endogenous ependymal region stem/progenitor cells following minimal spinal cord injury in the adult rat. Neuroscience 131:177-187

Okada S, Nakamura M, Katoh $\mathrm{H}$ et al (2006) Conditional ablation of Stat 3 or Socs 3 discloses a dual role for reactive astrocytes after spinal cord injury. Nat Med 12:829-834

Pfeifer K, Vroemen M, Blesch A, Weidner N (2004) Adult neural progenitor cells provide a permissive guiding substrate for corticospinal axon growth following spinal cord injury. Eur J Neurosci 20:1695-1704

Picard-Riera N, Nait-Oumesmar B, Baron-Van Evercooren A (2004) Endogenous adult neural stem cells: limits and potential to repair the injured central nervous system. J Neurosci Res 76:223-231

Rivieccio MA, John GR, Song X et al (2005) The cytokine IL-1beta activates IFN response factor 3 in human fetal astrocytes in culture. $\mathrm{J}$ Immunol 174:3719-3726

Samanta J, Kessler JA (2004) Interactions between ID and OLIG proteins mediate the inhibitory effects of BMP4 on oligodendroglial differentiation. Development 131:4131-4142
Shen AG, Liu YH, Zhao J et al (2008) Temporal-spatial expressions of p27kip1 and its phosphorylation on serine-10 after acute spinal cord injury in adult rat: implications for post-traumatic glial proliferation. Neurochem Int 52:1266-1275

See J, Zhang X, Eraydin N et al (2004) Oligodendrocyte maturation is inhibited by bone morphogenetic protein. Mol Cell Neurosci 26: 481-492

Sekhon LH, Fehlings MG (2001) Epidemiology, demographics, and pathophysiology of acute spinal cord injury. Spine 26:S2-S12

Sergent-Tanguy S, Michel DC, Neveu I, Naveilhan P (2006) Long-lasting coexpression of nestin and glial fibrillary acidic protein in primary cultures of astroglial cells with a major participation of nestin $(+) /$ GFAP(-) cells in cell proliferation. J Neurosci Res 83:1515-1524

Silver J, Miller JH (2004) Regeneration beyond the glial scar. Nat Rev Neurosci 5:146-156

Vargha-Khadem F, Gadian DG, Copp A, Mishkin M (2005) FOXP2 and the neuroanatomy of speech and language. Nat Rev Neurosci 6:131-138

Vartak-Sharma N, Ghorpade A (2012) Astrocyte elevated gene-1 regulates astrocyte responses to neural injury: implications for reactive astrogliosis and neurodegeneration. J Neuroinflammation 9:195

Weiss S, Dunne C, Hewson J et al (1996) Multipotent CNS stem cells are present in the adult mammalian spinal cord and ventricular neuroaxis. J Neurosci 16:7599-7609

White RE, McTigue DM, Jakeman LB (2010) Regional heterogeneity in astrocyte responses following contusive spinal cord injury in mice. $\mathrm{J}$ Comp Neurol 518:1370-1390

Wu J, Pajoohesh-Ganji A, Stoica BA, Dinizo M, Guanciale K, Faden AI (2012) Delayed expression of cell cycle proteins contributes to astroglial scar formation and chronic inflammation after rat spinal cord contusion. J Neuroinflammation 9:169

Yin X, Yin Y, Cao FL, Chen YF, Peng Y, Hou WG, Sun SK, Luo ZJ (2012) Tanshinone IIA attenuates the inflammatory response and apoptosis after traumatic injury of the spinal cord in adult rats. PLoS One 7:e38381

Zhang JL, Li DB, Shen AG et al (2013) Expression of RBMX after spinal cord injury in rats. J Mol Neurosci 49:417-429

Zhao J, Zhang S, Wu X et al (2011) KPC1 expression and essential role after acute spinal cord injury in adult rat. Neurochem Res 36:549-558 